\title{
Efeito hipotensor subagudo de uma sessão de treinamento intervalado de alta intensidade (HIIT)
}

\section{subacute hypotensor effect of a high intensity interval training session}

\author{
Gabriel Dutra de Jesus Siqueira, ${ }^{1}$ Leonardo Lousa Maioni, ${ }^{1}$ Viviane Soares, ${ }^{2}$ William Alves Lima \\ 'UniEVANGÉLICA, Anápolis, GO, Brasil. \\ ¿Universidade Federal de Goiás, Goiânia, GO, Brasil.
}

Recebido em: 05/12/2016 / Aceito em: 21/02/2017 / Publicado em: 31/03/2017

siqueiragdj@gmail.com

\section{RESUMO}

Objetivo: verificar a resposta da pressão arterial (no decorrer de 24h) após uma sessão de HIIT com o protocolo Tabata, bem como o comportamento da frequência cardíaca nesse período. Método: foram avaliados 17 indivíduos de ambos os sexos (seis homens e $11 \mathrm{mu}-$ Iheres) com idades entre 16 e 37 anos, que realizaram o protocolo de HIIT Tabata em cicloergômetro, sendo mensurada a pressão arterial e a frequência cardíaca dos mesmos, em repouso, após o aquecimento, logo após a realização do protocolo, após cinco, dez, 20, $30,40,50$ e 60 minutos e 24 horas. Os dados obtidos foram comparados através da ANOVA para medidas repetidas, e para determinar e avaliar as diferenças foi utilizado o post hoc de Bonferroni, adotando uma significância de $p \leq 0,05$. Resultados: foi identificado que há uma resposta hipotensora da pressão arterial sistólica a partir de 40 minutos, porém sem diferenças em 24 horas, sem diferenças significantes para diastólica, com o comportamento da frequência cardíaca dentro do esperado no período pós-HIIT. Considerações finais: no presente estudo, o HIIT gerou resposta hipotensora a partir de 40 minutos, após o término de sua execução e essa se mantem constante até o período de 60 minutos na pressão arterial sistólica. Este comportamento não foi encontrado na pressão arterial diastólica e, a frequência cardíaca, apresentou o comportamento de acordo com o esperado para outros protocolos de esforço físico moderado a intenso.

Palavras-chave: Educação Física e Treinamento, Pressão Arterial; Frequência Cardíaca.

\section{ABSTRACT}

Objective: verify the blood pressure response (during 24h) after a session of high intensity interval training (HIIT) with the Tabata protocol, as well as the heart rate behavior in this period. Method: we evaluated 17 individuals of both genders (six men and 11 women) aged 16 to 37 years, who performed the HIIT Tabata protocol on a cycle ergometer. Blood pressure and heart rate was measured at rest after heating, immediately after the protocol, after five, ten, 20, 30, 40, 50 and 60 minutes and 24 hours. The data obtained were compared through ANOVA for repeated measures, and the Bonferroni post hoc was used to determine and evaluate the differences, adopting a significance level of $p \leq 0.05$. Results: it was identified that there is a hypotensive response of systolic blood pressure starting in 40 minutes, but without differences in 24 hours, without significant differences for diastolic, with heart rate behavior within the expected period after HIIT. Closing remarks: in the present study HIIT generated a hypotensive response starting in 40 minutes after the end of its execution and this is maintained constant up to 60 minutes in the systolic blood pressure. This behavior was not found in diastolic blood pressure and heart rate presented behavior as expected for other protocols of moderate to intense physical effort.

Keywords: Physical Education and Training; Blood Pressure; Heart Rate.

\section{INTRODUÇÃO}

Pressão sanguínea corresponde à força que o sangue exerce contra qualquer parede de vasos, gerando uma pressão ou tensão nos mesmos. ${ }^{1}$ A Pressão Arterial possui um comportamento inconstante, durante o dia e varia de acordo a necessidade de maior ou menor tensão nos vasos, que relacionado ao comportamento físico, como parado, andando, correndo e emocional. 
A prática sistematizada de exercícios físicos é um fator que pode influenciar o comportamento da Pressão Arterial. Exercícios físicos têm ação comprovadamente positiva nos quadros clínicos de hipertensão arterial, com a atenção de que as dosagens de volume e intensidade do exercício podem influenciar diretamente nessa resposta, ${ }^{2}$ porém a resposta de cada protocolo de treinamento é especifica, entretanto, ainda não estão bem esclarecidas na literatura. O efeito hipotensor dos exercícios em geral é bem demonstrado mesmo em exercícios de baixa intensidade, em formas de circuito, ${ }^{3}$ ginástica localizada ${ }^{4}$ ou com a utilização de treino aeróbio seguido de treinamento de forç $\mathrm{a}^{5}$ e também com exercícios de cíclicos de resistência e de força. ${ }^{6}$ Ferreira et al. ${ }^{7}$ destacam que as regulações da pressão arterial decorrentes do exercício físico são importantes; mesmo que essas sejam mínimas, podem influenciar na sobrevida cardiovascular e são capazes de proteger os indivíduos, quanto ao desenvolvimento de doenças do sistema cardiovascular. Sendo o exercício físico um aliado que pode ser utilizado, tanto na prevenção, quanto no tratamento da hipertensão arterial sistêmica considerando que esta atinge a uma notória parcela da população e apresenta riscos de morbidade e mortalidade cardiovasculares. ${ }^{8}$

Trabalhos de alta intensidade sempre apresentam indagações quanto aos seus risco e benefícios. Entre as formas de métodos de trabalhos de alta intensidade, o Treinamento Intervalado de Alta Intensidade (HIIT) vem tomando cada vez mais adeptos, dentro das práticas regulares de exercícios físicos, tendo em vista os benefícios gerados e relacionados a mudanças na composição corporal, condicionamento físico aeróbio e anaeróbio, redução da gordura visceral e melhoras cardiovasculares. ${ }^{9}$ Contudo, há a preocupação de se investigar os efeitos agudos, subagudos e crônicos desse tipo de treinamento, atentando que este tem uma carga de trabalho de grande exigência mecânica e cardiocirculatória, apesar de ser realizado em curto período. ${ }^{9}$

O HIIT pode ser utilizado, tanto para o treinamento do público fitness, quanto para a preparação desportiva, sem que sejam afetadas as habilidades e técnicas da modalidade praticada, apenas deve ser atentado para o não abandono das especificidades e o cuidado com as proporções de cargas de treino de forma adequada e bem estruturada. ${ }^{10}$ Dessa forma, analisando o possível potencial de o HIIT poder influenciar diretamente no condicionamento físico geral de seu praticante, faz-se necessária a investigação de outros parâmetros que estão relacionados ao condicionamento físico, como a aptidão cardiocirculatória.

Atentando à relação entre exercício físico e controle da pressão arterial, fica em aberta a indagação de como e quanto o HIIT pode influenciar na pressão arterial. Além disso, como nos dias atuais a falta de tempo é um fator que atinge a grande parte da população. A crescente popularização do HIIT, talvez tenha se dado também por conta disso, visto os resultados e adaptação que o mesmo pode promover com sua realização em curto espaço de tempo. O "Tabata" talvez seja o protocolo de HIIT mais utilizado em academias, sendo caraterizado pela execução de apenas quatro minutos de treinamento intervalado.

A aplicação do HIIT, relacionada ao controle da pressão arterial parece estar ligada ao controle do sistema circulatório, pois sabe-se que exercícios de potência anaeróbia tem função importante no controle da pressão arterial. ${ }^{11}$ Essa melhora da potência anaeróbia foi demonstrada em um estudo de Tabata et al., ${ }^{12} \mathrm{em}$ que se compararam o treinamento de intensidade moderada de exercícios cíclicos de resistência e o HIIT, na melhora da capacidade anaeróbia e no $\mathrm{VO}_{2 \text { máx }}$ durante seis semanas. O estudo mostrou ainda que o treinamento com intensidade moderada não induz à melhora na capacidade anaeróbia, enquanto o HIIT é capaz de influenciar, tanto na melhora do $\mathrm{VO}_{2 \text { máx }}$ quanto na capacidade anaeróbia.

Cabe ressaltar que os protocolos de alta intensidade exigem certo nível de condicionamento físico do praticante. Dessa forma, o HIIT deve ser bem planejado e adequado pelos profissionais que irão realizar a prescrição do mesmo, conforme os objetivos dos indivíduos, suas potencialidades e limitações, e levando em consideração que trabalhos de alta intensidade têm seus riscos físicos, metabólicos, cardiovasculares, entre outros. ${ }^{13}$

Assim o objetivo do presente estudo foi verificar a resposta da pressão arterial sistêmica, após uma sessão de HIIT, com o protocolo Tabata, bem como a comportamento da frequência cardíaca nesse período.

\section{MÉTODO}

Trata-se de um estudo transversal realizado com indivíduos treinados (pelo menos seis meses de prática de musculação) de um estúdio de treinamento personalizado de Anápolis. 25 indivíduos, que já tinham experiência em realizar o protocolo Tabata em cicloergômetro, aceitaram o convite a participar do estudo. Houve perda amostral de oito pessoas, sendo então a amostra total da pesquisa de 17 voluntários normotensos de ambos os sexos (seis homens e 11 mulheres), com idades entre 16 e 37 anos (média de idade de 27,53 $\pm 6,35$ anos), a média de massa corporal de $68,39 \pm 12,91 \mathrm{~kg}$ e de estatura $1,69 \pm 7,86 \mathrm{~m}$.

Inicialmente, foi realizado o convite aos participantes do estudo. Após o aceite, os mesmos assinaram o Termo de Consentimento Livre e Esclarecido (TCLE), com as informações referentes à avaliação, conforme a Resolução 466 do ano de 2012 do Conselho Nacional de Saúde, sobre pesquisas com seres humanos, e a Declaração de Helsinque, de 1975. Depois disso, foram iniciados os procedimentos do protocolo de intervenção.

Para a mensuração de massa corporal e estatura foi utilizada uma balança da marca Welmy (R-110, Santa Bárbara d' Oeste, Brasil) e um estadiômetro de parede da marca WCS.

Os indivíduos foram classificados como normotensos de acordo com a sexta Diretriz Brasileira de Hipertensão, ${ }^{14}$ que classifica como hipertensas, pessoas que apresentam valores da Pressão Arterial sistólica igual ou superiores a $140 \mathrm{mmhg}$ e/ou da Pressão Arterial diastólica igual ou superiores a $90 \mathrm{mmhg}$.

Para mensuração a pressão arterial sistêmica (PA) e os valores da frequência cardíaca (FC) foi utilizado um monitor de pressão arterial automático Omron (HEM7200, Kyoto, Japão). A primeira medida foi realizada em repouso, aós o avaliado ficar cinco minutos sentado, a 


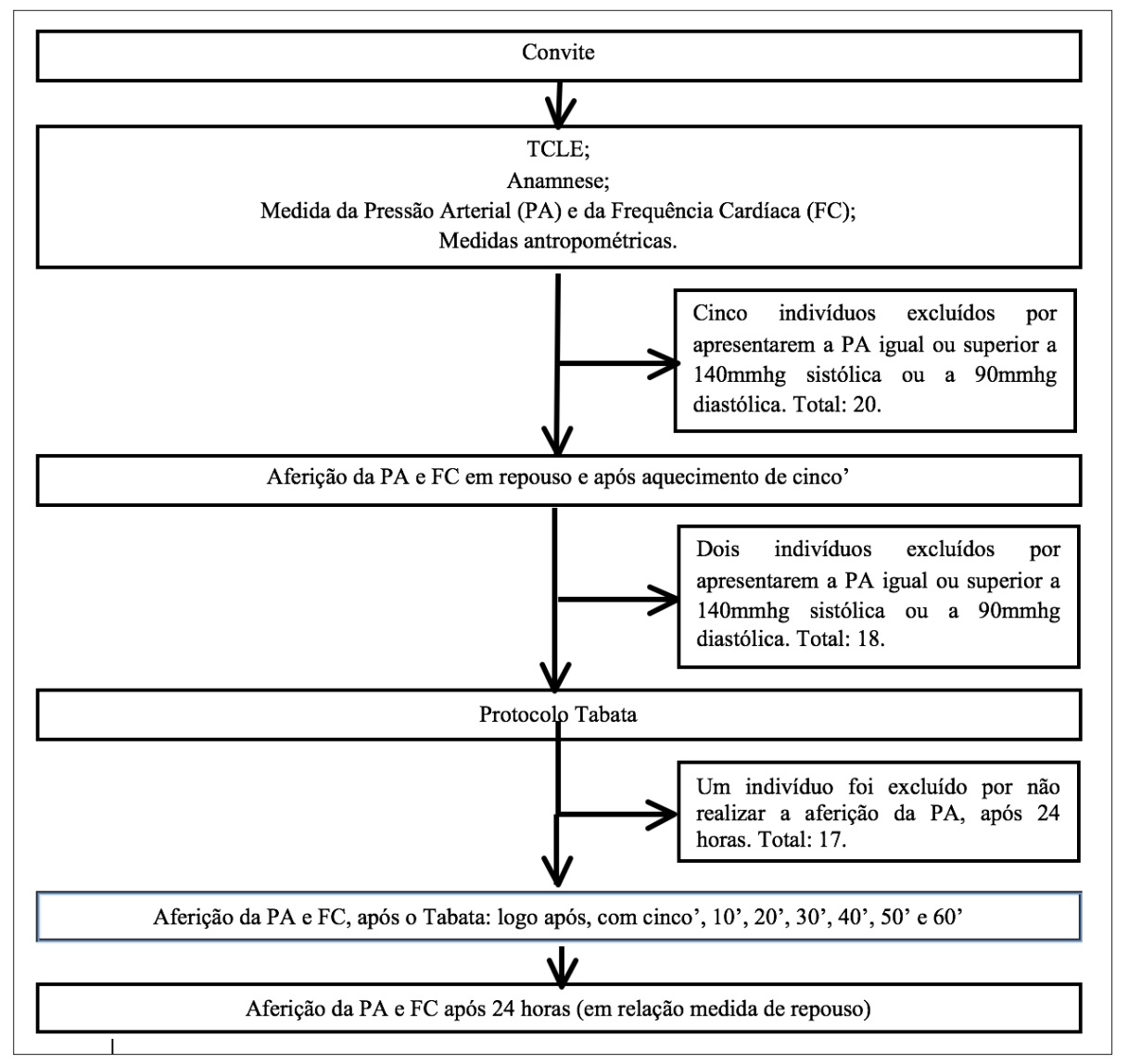

Figura 1 - Diagrama do estudo. Onde: '=minutos.

segunda medida foi realizada com o avaliado já posicionado na bicicleta (Keiser R3, Fresno, Estados Unidos), após o aquecimento de cinco minutos, antes de ser iniciado o protocolo Tabata. As demais medidas foram feitas logo após o protocolo e, subsequentemente, com cinco minutos, dez, 20, 30, 40, 50 e 60 minutos e 24 horas (em relação à medida de repouso); após o término do HIIT (estas medidas também realizadas com o indivíduo sentado, com repouso mínimo de cinco minutos), como representado na Figura 1, para melhor esclarecimento. Apenas a medida de PA e FC realizada após o aquecimento foi realizada com o voluntário sentado na bicicleta. As demais medidas foram realizadas com o avaliado sentado com postura ereta, joelhos flexionados a 90 graus sem cruzar as pernas, a medida foi realizado com o manguito fixado sempre no braço esquerdo dos indivíduos, este braço que ficou mantido em apoio à altura do coração, durante a medida.

O protocolo do HIIT realizado foi iniciado com o aquecimento de cinco minutos na bicicleta com o padrão de carga dez (a carga na referida bicicleta pode ser escalonada de um a 24, o que representou pouco mais que $40 \%$ da carga máxima da bicicleta) e manutenção de rotações acima de 70 RPM. Após isso, o procedimento adotado foi o de se alternar a realização de 20 segundos de intensidade alta, estabelecendo uma carga máxima em que o avaliado conseguisse a manutenção das rotações acima de 100 RPM, com ajuste de carga conforme a diminuição deste número de rotações, com intervalos de dez segundos, com carga mínima, sendo oito séries deste trabalho, totalizando quatro minutos. ${ }^{12}$
O valor de RPM foi obtido do próprio cicloergômetro que fornece o mesmo em seu painel, bem como o controle de carga que é visualizado também no painel e foi dosada pelo avaliador, durante a realização do protocolo.

Os dados foram expressos como média e desvio-padrão. Para verificar a distribuição das variáveis foi utilizado o teste de Sahpiro-Wilk. Para verificar diferenças entre as medidas de PA e FC foi utilizado à análise de variância (ANOVA) para medidas repetidas com post hoc de Bonferroni. O valor de $\mathrm{p}$ considerado foi $\leq 0,05$. Todas essas estatísticas foram analisadas no software SPSS para Windows versão 21.0 .

\section{RESULTADOS}

$\mathrm{Na}$ tabela 1 estão apresentados os valores gerais das medidas da pressão arterial e da frequência cardíaca momento a momento, comparando o valor de cada período com o respectivo subsequente, nos períodos repouso, antes, logo após, cinco, dez, 20, 30, 40, 50, 60 minutos e 24 horas após, com média e desvio padrão. Quanto ao comportamento da pressão arterial sistólica foram encontradas diferenças entre os períodos: repouso e antes $(p=0,010)$; antes e logo após $(p<0,001)$; logo após e cinco minutos após $(p<0,001)$; cinco minutos após e dez minutos $(p=0,001)$; dez minutos e 20 minutos $(p=0,002)$; e entre 60 minutos e 24 horas $(\mathrm{p}=0,003)$. Quanto à pressão arterial diastólica, só houve diferença entre logo após e cinco minutos após $(p<0,001)$. Já a frequência cardíaca apresentou dife- 
Tabela 1 - Comparação momento a momento das medidas da PS, PD e FC em média e DP no diferentes períodos de mensuração.

\begin{tabular}{|c|c|c|c|c|c|c|c|c|c|}
\hline \multirow{2}{*}{$\begin{array}{l}\text { Medidas } \\
\text { Tempo }\end{array}$} & \multicolumn{3}{|c|}{ PS (mmhg) } & \multicolumn{3}{|c|}{ PD (mmhg) } & \multicolumn{3}{|c|}{ FC (bpm) } \\
\hline & Média & DP & $\mathbf{p}$ & Média & DP & $\mathbf{p}$ & Média & DP & p \\
\hline \multirow[t]{2}{*}{ Repouso } & 120,18 & 13,11 & & 69,18 & 10,57 & & 72,88 & 13,14 & \\
\hline & & & $0,010^{*}$ & & & 0,327 & & & $<0,001 *$ \\
\hline \multirow[t]{2}{*}{ Antes } & 130,24 & 17,26 & & 71,82 & 13,24 & & 88,94 & 14,94 & \\
\hline & & & $<0,001 *$ & & & 0,059 & & & $<0,001 *$ \\
\hline \multirow[t]{2}{*}{ Logo Após } & 160,35 & 18,40 & & 78,00 & 10,49 & & 147,12 & 12,27 & \\
\hline & & & $<0,001^{*}$ & & & $<0,001^{*}$ & & & $<0,001^{*}$ \\
\hline \multirow[t]{2}{*}{ Após 5' } & 134,29 & 20,55 & & 67,41 & 10,06 & & 116,29 & 13,09 & \\
\hline & & & 0,001 * & & & 0,519 & & & $0,001 *$ \\
\hline \multirow[t]{2}{*}{ Após 10' } & 123,59 & 11,51 & & 66,06 & 7,09 & & 111,88 & 12,79 & \\
\hline & & & $0,002^{*}$ & & & 0,709 & & & $<0,001^{*}$ \\
\hline \multirow[t]{2}{*}{ Após 20' } & 116,24 & 10,47 & & 65,47 & 9,37 & & 106,00 & 13,29 & \\
\hline & & & 0,108 & & & 0,594 & & & $0,004^{*}$ \\
\hline \multirow[t]{2}{*}{ Após 30' } & 113,59 & 12,59 & & 66,06 & 8,63 & & 102,53 & 13,60 & \\
\hline & & & 0,099 & & & 0,491 & & & $<0,001 *$ \\
\hline \multirow[t]{2}{*}{ Após 40' } & 110,00 & 9,92 & & 64,24 & 11,44 & & 95,47 & 13,42 & \\
\hline & & & 0,785 & & & 0,596 & & & $0,003^{*}$ \\
\hline \multirow[t]{2}{*}{ Após 50' } & 110,53 & 10,27 & & 65,65 & 8,72 & & 91,18 & 13,15 & \\
\hline & & & 0,349 & & & 0,863 & & & $0,025^{*}$ \\
\hline \multirow[t]{2}{*}{ Após 60' } & 111,94 & 9,75 & & 65,88 & 7,94 & & 88,82 & 14,57 & \\
\hline & & & $0,003 *$ & & & 0,188 & & & $<0,001^{*}$ \\
\hline Após 24H & 116,12 & 9,91 & & 67,53 & 8,71 & & 71,94 & 12,49 & \\
\hline
\end{tabular}

Onde: $P S=$ Pressão Arterial Sistólica; $P D=$ Pressão Arterial Diastólica; $F C=$ Frequência Cardíaca; $D P=$ Desvio Padrão; Antes = refere-se ao momento pós-aquecimento, antes do início do protocolo; ' = minutos; $24 \mathrm{H}=$ vinte e quatro horas; $e^{*}=$ significante para $p \leq 0,05$ em comparação ao período anterior.

rença em seu comportamento entre todas as medidas.

Na figura 2 é mostrado o comportamento da pressão arterial nos diferentes períodos de mensuração, em que esses foram comparados com o período de repouso (cinco minutos antes), sente este o período que melhor representa a pressão arterial normal do cotidiano dos avaliados, que representaria uma espécie de controle do voluntário em relação a ele mesmo. Foram encontradas diferenças significativas para: o comportamento de elevação da pressão arterial sistólica, logo após o HIIT em relação ao período de repouso $(p<0,001)$; as demais diferenças, quanto à pressão arterial sistólica ocorreram apenas no grupo geral, acontecendo à resposta hipotensora nos períodos de $40(p=0,10), 50(p=0,017)$ e $60(p=0,015)$ minutos em comparação ao repouso; e não houve nenhuma diferença estatística para a pressão arterial diastólica.

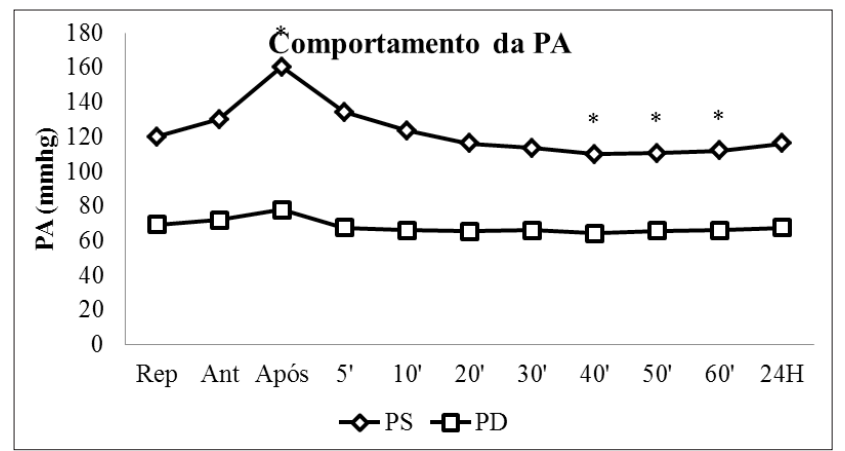

Onde: $P A=$ Pressão Arterial; $R e p=$ repouso; Ant = antes do início do protocolo, após o aquecimento; Após = logo após a finalização da aplicação do protocolo; $'=$ minutos $; 24 \mathrm{H}=$ vinte e quatro horas; $P S=$ Pressão Sistólica; $P D=$ Pressão Diastólica; $e^{*}=$ significativo para $p \leq 0,05$ em relação ao repouso.

Figura 2 - Distribuição e comparação do comportamento da Pressão Arterial (sistólica e diastólica) nos diferentes períodos mensurados.
Na figura 3 é apresentado o comportamento da frequência cardíaca, que se mostrou adequada, quanto aos períodos de exercício e repouso posterior, principalmente, em relação ao repouso, que não apresentou diferenças com 24 horas o que era esperado.

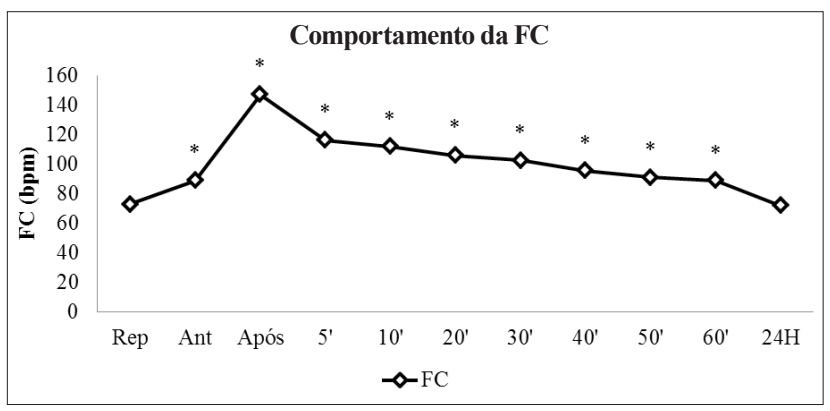

Onde: FC= Frequência Cardíaca; Rep = repouso; $A n t=$ antes do início do protocolo, após o aquecimento; Após = logo após a finalização da aplicação do protocolo; '= minutos; $24 \mathrm{H}=$ vinte e quatro horas; $e^{*}=$ significativo para $p \leq 0,05$ em relação ao repouso.

Figura 3 - Distribuição e comparação do comportamento da Frequência Cardíaca nos diferentes períodos mensurados.

\section{DISCUSSÃO}

O objetivo do presente estudo foi verificar a resposta da pressão arterial sistêmica após uma sessão de HIIT com o protocolo Tabata, bem como a comportamento da frequência cardíaca nesse período, de forma que os resultados esperados eram que a pressão arterial se elevasse durante o HIIT e em seguida caísse gradativamente, gerando o efeito hipotensor em relação 
ao período inicial de repouso e que a frequência cardíaca se comportasse da mesma forma. Os resultados sugeriram que há a resposta hipotensora da pressão arterial sistólica, sem diferenças para diastólica e comportamento adequado para a frequência cardíaca, no período pós-HIIT.

Um estudo realizado com militares de diferentes níveis de condicionamento, em que foi aplicado um protocolo de treinamento intervalado aeróbio de corrida, houve a resposta hipotensiva subaguda constante da pressão arterial diastólica, diferente do presente estudo que não apresentou resposta hipotensora na pressão diastólica, já, em relação à pressão sistólica e média, foi apresentada hipotensão sistólica significante com 60 minutos, em relação à primeira medida em repouso; ${ }^{15}$ no presente estudo esse comportamento começou a ser demonstrado com 40 minutos, diferença que talvez seja justificada pelas diferenças de intensidades dos protocolos aplicados. Já, no estudo de Pardono et al., ${ }^{16}$ foi analisado o efeito hipotensor de corredores em dois protocolos diferentes, o primeiro de corrida máxima de 1600 metros e o segundo de corrida submáxima de 20 minutos na zona de $75-80 \%$ de intensidade equivalente. Foi achado que ambos as corridas foram capazes de gerar o efeito hipotensor, contudo no período de 45 minutos, a magnitude da hipotensão do grupo que realizou o protocolo máximo foi significante superior em relação ao grupo controle e também em relação ao grupo submáximo. No presente estudo, o protocolo realizado também exigiu alta intensidade para o seu período de realização, e a resposta hipotensora ocorreu a partir dos 40 minutos, período similar ao do estudo citado.

Osawa et al. ${ }^{17}$ realizaram um estudo que avaliou os efeitos de 16 semanas de HIIT, utilizando ergômetros de membros superiores e inferiores. Dentre os resultados do estudo, foi mostrado que apenas o grupo que realizou o HIIT em cicloergômetro melhorou o $\mathrm{VO}_{2 \text { pico }}$ i o outro grupo, que realizou o HIIT em ergômetros de membros superiores e também no cicloergômetro, não obtiveram essa melhora de forma significativa. No presente estudo, também foi utilizado o cicloergômetro para a execução, e notando a resposta hipotensora obtida esse tipo de ergômetro parece ser bastante efetivo por conseguir atingir a diferentes tipos de adaptações. Rosa et al. ${ }^{11}$ aplicaram o protocolo de HIIT denominado Wingate, em sedentários com o intuito de avaliar a resposta hipotensiva aguda de um exercício de potência anaeróbia e realizaram as medidas da pressão arterial em repouso, após o teste, com 30, 60, 90 e 120 minutos. Nos resultados foi encontrada diferença significante para hipotensão com 120 minutos. O protocolo Tabata aplicado no presente também apresenta essa característica de potência anaeróbia, contudo a hipotensão aconteceu em período menor pós-exercício em relação ao estudo citado. Talvez esse fato possa ser explicado pelo fato de que os voluntários do presente estudo já eram treinados, adaptados ao protocolo Tabata e, habituados ao cicloergômetro utilizado.

Em um estudo ${ }^{18}$ realizado, que utilizou ratos como amostra, foi demonstrado que em um protocolo de treinamento intervalado que se utiliza de intensidades mais altas, alternando com períodos de descanso, ocorre maior redução da frequência cardíaca e do duplo produto em comparação ao treinamento contínuo de intensidade moderada. No estudo de Carvalho et al. ${ }^{19}$ que avaliaram a resposta de magnitude e duração da hipotensão pós-exercício com idosos, comparando treinamento contínuo e intervalado, foi achado que ambos os treinamentos promovem a resposta hipotensora, considerando pressão arterial sistólica, diastólica média e duplo produto, no período de vinte e quatro horas seguintes a realização do exercício, sendo ressaltado que a magnitude do treinamento intervalado foi maior, além do que o mesmo promove menor sobrecarga cardiovascular. Outro estudo ${ }^{20}$ analisou um público de pacientes com síndrome metabólica e compararam treinamento contínuo e HIIT. Os pesquisadores demonstraram que ambos, conciliados a uma reeducação do estilo vida, apresentaram melhoras na diminuição de intervalos de recuperação e que o HIIT é capaz de promover melhoras mais efetivas em alguns fatores de risco cardiometabólicos. Vale ressaltar que além das comparações entre as formas e métodos de treinamento, é importante que o indivíduo se apodere dos diversos benefícios dos mesmos, como melhoras neuromusculares e de aptidão cardiorrespiratória, usufruindo de um possível efeito crônico do exercício físico, ${ }^{21}$ de forma que ele não fique atrelado ao mesmo treinamento por ser mais eficiente em determinado componente. Mas, relacionando os achados do presente estudo com os dados vigentes na literatura, trabalhos com maiores intensidades parecem ser mais efetivos quanto às respostas cardiovasculares, e por ser de curta duração, a sobrecarga cardíaca é relativamente pequena.

Emberts et al. ${ }^{22}$ realizaram um estudo que analisou o efeito da adição de mais rounds ao protocolo Tabata, utilizando exercícios com peso corporal, como os pliométricos, totalizando o tempo de 20 minutos de sessão de HIIT, somando tempos de trabalho e intervalos. Os dados encontrados no estudo sugeriram que essa sessão proposta pelos autores atende às diretrizes do ACSM para melhora na aptidão cardiorrespiratória, além do protocolo ter sido bem tolerado pelos avaliados, o que pode resultar em maior dispêndio calórico na sessão. No presente estudo o protocolo Tabata foi utilizado com o trabalho total de quatro minutos, e com apenas esse período de tempo se mostrou efetivo, quanto à resposta cardiovascular avaliada, sem sobrecarga excessiva.

Esse mesmo comportamento foi demonstrado em um estudo ${ }^{23}$ que utilizou o exercício "Kettlebell Swing", com a aplicação do protocolo Tabata, comparado ao treinamento tradicional desse exercício, que consistia em quatro séries de execução (sendo que foi contado o número de repetições realizadas no protocolo Tabata, e esse número foi dividido e distribuídos nessas quatro séries do protocolo tradicional) com noventa segundos de recuperação, foi achado que o protocolo Tabata promoveu respostas cardiovasculares e metabólicas mais significantes em magnitude, comparado ao protocolo tradicional utilizado.

No estudo de Lima et al. ${ }^{24}$ foi mostrado que em exercícios de força com intervalos mais curtos de recuperação, a frequência cardíaca se mantem mais alta durante a hora posterior a realização do exercício, apesar disso, essa resposta é apenas transitória e, com 24 horas as respostas cardiovasculares, independente do intervalo de descanso, são iguais. Esses dados vão ao encontro do que foi achado no presente estudo em 
relação à frequência cardíaca.

Lovato et al. ${ }^{25}$ identificaram uma redução da frequência cardíaca e da pressão arterial pós 24 horas da prática de exercícios aeróbios ou de exercícios resistidos, que duraram aproximadamente 60 minutos de estimulação. Justificam que a redução da pressão arterial pode ser em decorrência de uma diminuição da frequência cardíaca, com consequente redução do volume sistólico, o que ocorreu no presente estudo, apesar da frequência cardíaca não ter reduzido abaixo dos níveis de repouso, o que leva a busca de outros fatores para explicar tal redução. Sabe-se também que a pressão arterial pode reduzir devido a uma queda da resistência vascular sistêmica, pós o esforço cardíaco demandado com o exercício. ${ }^{26,27}$ Há também a possibilidade de ação de fatores humorais relacionados à produção de fatores vasoativos como o peptídeo natriurético atrial, que tem o papel de normalizar a volemia sanguínea e a pressão arterial quando a musculatura cardíaca foi excessivamente distendida, o que ocorre com a demanda circulatória em exercícios de alta intensidade, ${ }^{28}$ como o proposto na presente pesquisa.

Outro fator que pode contribuir para o efeito vasodilatador pós exercício são as possíveis alterações funcionais dos pressorreceptores arteriais e cardiopulmonares, levando a um possível aumento na sua sensibilidade e uma consequente modificação no seu ponto de ativação e do seu tempo de recuperação. Com isso, ocorre uma sensível redução na resposta vasoconstritora alfa-adrenérgica, verificada no período de recuperação dos receptores alfa-adrenérgicos, o que também poderia explicar o maior fluxo sanguíneo muscular pós-exercício. ${ }^{29}$ Contudo, não se pode desconsiderar a ação dos receptores beta adrenérgicos vasculares que estimulam a síntese de óxido nítrico endotelial, com a consequente oxidação de nitrogênio terminal, Uma vez formado, o óxido nítrico difunde-se rapidamente da célula endotelial para a célula muscular lisa, onde interage, estimulando a sua atividade catalítica e levando à diminuição dos níveis intracelulares de $\mathrm{Ca}^{2+}, 30,31$ o que levaria a uma vasodilatação.

\section{CONSIDERAÇÕES FINAIS}

No presente estudo, o HIIT foi capaz gerar resposta hipotensora a partir de 40 minutos após o término de sua execução e essa se manteve constante ate o período de 60 minutos na pressão arterial sistólica. Este comportamento não foi encontrado na pressão arterial diastólica e a frequência cardíaca apresentou normalmente seus períodos de elevação, queda após o exercício e sem diferenças após 24 horas.

Apesar dos achados, cabe-se ressaltar os fatores limitantes do presente estudo, como os fatos de não haver grupo controle e o tamanho amostral, fatores que poderiam deixar os resultados mais confiáveis. Sugere-se que este estudo seja replicado de forma crônica, avaliando esse efeito em hipertensos, com as adequações necessárias devido à condição física e fisiológica dos mesmos.

\section{REFERÊNCIAS}

1. Guyton AC, Hall JE. Tratado de fisiologia médica. Elsevier
Rio de Janeiro, 2011; 172 p.

2. Baganha RJ, de Paula CF, Vieira LM, Dias R, Oliveira LHS, de Souza e Silva A, daSilva Júnior AJ, Paiva Neto A. Hipertensão arterial sistêmica e exercício físico: adaptações e mecanismos hipotensores associados. RBPFEX-Revista Bras Prescrição e Fisiol do Exerc. 2014;8(47).

3. Miranda AR, Benini R, Nunes PRP, da Silva BVC, Neto OB, da Mota GR, Orsatti FL. Resposta pressórica pósexercício resistido em circuito de membros superiores e inferiores. RBPFEX-Revista Bras Prescrição e Fisiol do Exerc 2012;6(33):313-18.

4. Moura TNB de, Silva CMG da, Orsano VSM, Lopes F, Júnior S, Silva GCB. Hipotensão pós-exercício após uma sessão de exercícios físicos em idosas normotensas e hipertensas. Estud Interdiscip sobre o Envelhec 2012;17(2):281-84. doi: 10.1590/S1517-86922006000600003

5. Santiago DA, Moraes JFVN de, Mazzoccante RP, Boullosa DA, Simões HG, Campbell CSG. Corrida em esteira e exercícios de força: efeitos agudos da ordem de realização sobre a hipotensão pós-exercício. Rev Bras Educ Física e Esporte 2013;27(1):67-73. doi: 10.1590/S180755092013000100007

6. Macedo M, Silva A, Olher R, Coelho-Junior H, Palmeira $R$, Asano R. Post-exercise hypotension between different protocols of resistance training for beginners. J Exerc Physiol Online 2014;17(6):58-66. doi: 10.1590/S180759322011000200007

7. Ferreira AP, Campos BRM, Rodrigues Junior É, Puga GM, Brito CJ. Effects of aerobic and resistance exercise intensities on 24-hours blood pressure in normotensive women. Mot Rev Educ física 2013;19(4):681-7. doi: 10.1590/S198065742013000400003

8. Prado ALM, Silva CEB, Brito CP, Prado MCM, Prado OMP. Exercício físico no tratamento da hipertensão arterial sistêmica: Hipotensão pós-exercício e prescrição de exercício físico para pacientes hipertensos/Physical exercise in the treatment of hypertension: Post exercise hypotension and exercise prescription. Motricidade 2012;8(S2):719-24.

9. Kravitz L. ACSM information on high intensity interval training. 2015.

10. Herodek K, Simonović C, Pavlović V, Stanković R. High Intensity Interval Training. Act Phys Educ Sport 2014;4(2):205-7.

11. Rosa HI, Pantoja FL, Rabelo AS, Costa HA, Pires FO, Silva-Filho AC, Barros CLM, Puga GM, Mendes TT. Acute Hypotension Effect of a Single Bout of Anaerobic Power Exercise (30-Sec Wingate Test). J Exerc Physiol Online 2015;18(6):63-69.

12. Tabata I, Nishimura K, Kouzaki M, Hirai Y, Ogita F, Miyachi $M$, et al. Effects of moderate-intensity endurance and highintensity intermittent training on anaerobic capacity and VO2max. Med Sci Sports Exerc 1996;28(10):1327-30.

13. Olson M. TABATA: It'sa HIIT! ACSMs Health Fit J 2014;18(5):17-24.

14. Hipertensão VDB de. VI Diretrizes Brasileiras de Hipertensão. 2010.

15. de Sá MC, de Lira EB, Duarte AFA. Efeitos do treinamento intervalado na resposta hipotensiva de militares com diferentes padrões de condicionamento físico. Rev Bras Ciências do Esporte 2013;36(1):45-58. doi: 10.1590/S010132892014000100004

16. Pardono E, Fernandes $M$ de O, Azevêdo LM, Almeida JA de, Mota MR, Simões HG. Post-exercise hypotension of 
normotensive young men through track running sessions. Rev Bras Med do Esporte 2015;21(3):192-5. doi: 10.1590/1517869220152103137534

17. Osawa Y, Azuma K, Tabata S, Katsukawa F, Ishida H, Oguma Y, Kawai T, Itoh H, Okuda S, Matsumoto H. Effects of 16-week high-intensity interval training using upper and lower body ergometers on aerobic fitness and morphological changes in healthy men: a preliminary study. Open access $\mathrm{J}$ Sport Med 2014;4(5):257-65. doi: 10.2147/OAJSM.S68932

18. Borges JP, Masson GS, Tibiriçá E, Lessa MA. Exercício Aeróbio Intervalado Induz Maior Redução na Sobrecarga Cardíaca na Recuperação em Ratos. Arq bras cardiol 2014;102(1):47-53. doi: 10.5935/abc. 20130230

19. Carvalho RST de, Pires CMR, Junqueira GC, Freitas D, Marchi-Alves LM. Hypotensive Response Magnitude and Duration in Hypertensives: Continuous and Interval Exercise. Arq Bras Cardiol 2015;104(3):234-41. doi: org/10.5935/ abc. 20140193

20. Drigny J, Gremeaux V, Guiraud T, Gayda M, Juneau M, Nigam A. Long-term high-intensity interval training associated with lifestyle modifications improves QT dispersion parameters in metabolic syndrome patients. Ann Phys Rehabil Med 2013;56(5):356-70. doi: 10.1016/j.rehab.2013.03.005

21. Cunha FA, Matos-Santos L, Massaferri RO, Monteiro TPL, Farinatti PT V. Hipotensão pós-exercício induzida por treinamento aeróbio, de força e concorrente: aspectos metodológicos e mecanismos fisiológicos. Rev Hosp Univ Pedro Ernesto 2013;12(4)99-112. doi: 10.12957/ rhupe. 2013.8717

22. Emberts T, Porcari J, Dobers-tein S, Steffen J, Foster C. Exercise intensity and energy expenditure of a Tabata workout. J Sports Sci Med 2013;12(3):612-13.

23. Fortner HA, Salgado J, Holmstrup AM, Michael E. Cardiovascular and metabolic demands of the kettlebell swing using a Tabata interval versus a traditional resistance protocol. Int J Exerc Sci 2014;7(3):179-85.
24. Lima AHR de A, Forjaz CL de M, Silva GQ de M, Lima APA, Lins Filho OL, Cardoso Júnior CG, Ritti-Dias RM. Effect of rest interval on cardiovascular responses after resistance exercise. Motriz: Rev Educ Física 2013;19(2):252-60. doi: 10.1590/S1980-65742013000200002

25. Lovato NS, Anunciação PG, Polito MD. Pressão arterial e variabilidade de frequência cardíaca após o exercício aeróbio e com pesos realizados na mesma sessão. Rev Bras Med Esporte 2012;18(1):22-5. doi: org/10.1590/S151786922012000100004

26. Nami R, Mondillo S, Lenti S, Ferro G, Nami N, Tarantino M, Glauco G, Spanò E, Gennari C. Aerobic exercise training fails to reduce blood pressure in nondipper-type hypertension. Am J Hypertens 2000;13(6):593-600.

27. Legramante JM, Galante A, Massaro M, Attanasio A, Raimondi G, Pigozzi $F$, et al. Hemodynamic and autonomic correlates of postexercise hypotension in patients with mild hypertension. Am J Physiol Integr Comp Physiol 2002;282(4):1037-43. doi: 10.1152/ajpregu.00603.2001

28. Negrão CE, Rondon M. Exercício físico, hipertensão e controle barorreflexo da pressão arterial. Rev Bras Hipertens 2001;8(1):89-95.

29. de Moraes Forjaz CL, Santaella DF, Rezende LO, Barretto ACP, Negrão CE. A duração do exercício determina a magnitude e a duração da hipotensão pós-exercício. Arq Bras Cardiol 1998;70(2):99-104.

30. Dessy C, Moniotte S, Ghisdal P, Havaux X, Noirhomme $P$, Balligand J-L. Endothelial $\beta 3$-adrenoceptors mediate vasorelaxation of human coronary microarteries through nitric oxide and endothelium-dependent hyperpolarization. Circulation 2004;110(8):948-54. doi: 10.1161/01. CIR.0000139331.85766.AF

31. Murad F. The nitric oxide-cyclic GMP signal transduction system for intracellular and intercellular communication. Recent Prog Horm Res 1993;49:239-48.

Como citar: SIQUEIRA, Gabriel Dutra de Jesus et al. Efeito hipotensor subagudo de uma sessão de treinamento intervalado de alta intensidade (HIIT). Cinergis, Santa Cruz do Sul, v. 18, n. 2, mar. 2017. ISSN 2177-4005. Disponível em: <https://online.unisc.br/ seer/index.php/cinergis/article/view/8687>. Acesso em: 29 mar. 2017. doi:http://dx.doi.org/10.17058/cinergis.v18i2.8687. 